\title{
OVARIECTOMY UP-REGULATES NEURONAL NEUROFILAMENT LIGHT CHAIN mRNA EXPRESSION WITH REGIONAL AND TEMPORAL SPECIFICITY
}

\author{
E. VAUCHER, ${ }^{a} \dagger$ P. PIERRET, ${ }^{\mathrm{a}}$ J.-P. JULIEN ${ }^{\mathrm{a}}$ and G. A. KUCHEL ${ }^{\mathrm{b}}{ }^{\mathrm{a}}$ \\ ${ }^{a}$ Centre for Research in Neuroscience, Montreal General Hospital and McGill University, 1650 Cedar Avenue, Montreal, \\ Quebec, H3G 1A4, Canada \\ bUConn Center on Aging, MC-5215, University of Connecticut Health Center, 263 Farmington Avenue, Farmington,
} CT 06030-5215, USA

\begin{abstract}
Estrogens can influence the survival, plasticity and function of many adult neurons. Many of these effects, such as neurite outgrowth and increased dendritic spine density, are mediated by changes in neuronal cytoskeletal architecture. Since neurofilament proteins play a key role in the maintenance and remodeling of the neuronal cytoskeleton, we postulated that changes in neurofilament light chain mRNA may parallel some of the alterations in neuronal architecture which follow bilateral ovariectomy. We measured neurofilament light chain mRNA levels using a ribonuclease protection assay at two time-points after ovariectomy in mature female rats. One week after ovariectomy, neurofilament light chain mRNA levels (corrected for glucose-6-phosphate dehydrogenase mRNA) did not differ from sham-operated animals in the five brain regions examined (hypothalamus, striatum, hippocampus, frontal cortex and occipital cortex). Four months after ovariectomy, neurofilament light chain mRNA levels were similarly unchanged in the hypothalamus and striatum. In contrast, statistically significant increases in neurofilament light chain mRNA expression were observed in the three regions receiving basal forebrain projections (hippocampus, frontal cortex and occipital cortex). In situ hybridization demonstrated increases in neurofilament light chain mRNA expression involving subpopulations of smaller medial septal neurons. There also appeared to be an increased number of larger septal neurons following long-term ovariectomy.

We propose that atrophic changes involving basal forebrain projection fibers are followed by compensatory axonal growth by other 'intact' basal forebrain neurons. Increased neurofilament light chain mRNA expression and somatic hypertrophy in medial septal neurons may both be reflective of the need to sustain an axonal network which is larger and more complex. In contrast, increased neurofilament light chain mRNA expression observed in basal forebrain targets following long-term ovariectomy may be reflective of compensatory changes taking place in local neurons. (C) 2001 IBRO. Published by Elsevier Science Ltd. All rights reserved.
\end{abstract}

Key words: aging, Alzheimer's disease, axonal cytoskeleton, basal forebrain, hormone replacement therapy, in situ hybridization.

The female menopause is characterized by a loss of ovaries as a source of estrogen and progesterone production, resulting in significant declines in levels of these hormones in many older women. ${ }^{24}$ Hormone replacement therapy (HRT) with estrogen has been shown to be useful in the prevention of age-related events such as peri- and post-menopausal bone loss. Several recent studies indicate that women receiving HRT tend to have lower rates of Alzheimer's disease (AD) ${ }^{18,53}$ than their matched controls. In addition, gonadal steroids (GS) can influence the ability of many cells, including some neuronal populations, to maintain normal structure and

*Corresponding author. Tel.: +1-860-679-3959; fax +1-860-6791307.

E-mail address: kuchel@ns01.uchc.edu (G. A. Kuchel).

$\dagger$ Present address: School of Optometry, Université de Montréal, Montreal, Quebec, Canada.

Abbreviations: AD, Alzheimer's disease; ChAT, choline acetyltransferase; GAPDH, glucose-6-phosphate dehydrogenase; GS, gonadal steroids; HRT, hormonal replacement therapy; LI, labeling index; NF-L, neurofilament light chain; NGF, nerve growth factor; OVx, ovariectomy; SSC, saline sodium citrate. function. As a result, there has been a growing interest in exploring the possibility that HRT may prevent AD or, at least, slow its progression. Although HRT can restore cognitive deficits induced by ovariectomy (OVx) in rodents, most studies of older women have so far been negative. ${ }^{12}$ The above considerations highlight the need to examine the longer term consequences of $\mathrm{OVx}$, particularly as they impact upon the cytoskeleton, since this could produce alterations in neuronal architecture, possibly even resulting in lost neuronal connectivity and in the formation of pathological structures. Estrogens can modulate the cytoskeletal architecture of susceptible neurons, since both dendritic spine density in hippocampal pyramidal neurons in rodents, ${ }^{16,40}$ as well as outgrowth of neurites in cell cultures ${ }^{5,6,31}$ have been shown to increase as a function of higher estrogen levels.

$\mathrm{AD}$, the most common neurodegenerative cause of dementia in old age, is characterized primarily by a degeneration of cholinergic neurons in the basal forebrain. As a result, a cholinergic deficit develops in relevant projection areas, particularly the neocortex and the hippocampus, with resultant cognitive deficits involving 
attention, learning and memory. Interestingly, OVx in rodents impairs cholinergic function ${ }^{15,33}$ and GS replacement, especially with $17 \beta$-estradiol administration, tends to alleviate the negative effects of $\mathrm{OVx}$ on cholinergic parameters as well as on cognitive performance involving a number of learning and memory tasks. ${ }^{37,44}$ The precise mechanisms leading to the profound, yet highly specific, neurodegeneration observed in $\mathrm{AD}$ have not yet been determined. Nevertheless, one of the cardinal pathological features of AD is a neurofibrillary anomaly consisting of the accumulation of neurofilament proteins ${ }^{50}$ in the neuronal perikaryon, ${ }^{19}$ in dystrophic neurites within neuropil threads, ${ }^{57}$ in neuritic plaques ${ }^{11,42,57}$ and in neurofibrillary tangles. ${ }^{56,60}$ Neurofilaments are heteropolymers composed of three proteins, a major component of the axonal cytoskeleton being the neurofilament light chain protein (NF-L, $68 \mathrm{kDa})$. Intracellular accumulation of NF-L protein as a result of NF-L overexpression in transgenic mice produces specific pathologic changes with perykarial swelling, as well as neurodegeneration in the spinal cord ${ }^{62}$ and in specific brain areas $^{34}$ (for review see Ref. 26). In contrast to the accumulation of neurofilament proteins, NF-L mRNA levels appear to be decreased in brains affected by $\mathrm{AD},{ }^{10,35}$ in other neurodegenerative diseases, ${ }^{26}$ as well as in normal aging. ${ }^{28,46}$

We previously showed that the development of axonal atrophy in aged sympathetic neurons was associated with decreased NF-L mRNA expression, and proposed that modulation in NF-L expression could be one of the primary events leading to axonal atrophy and possibly degeneration. ${ }^{28}$ In order to determine whether the deleterious effects of post-menopausal ovarian hormone deficiency on neuronal function could be mediated through modification of the cytoskeletal neuronal structure, we examined the effects of bilateral OVx on NF-L mRNA expression in specific rat brain regions. We used a ribonuclease protection assay and in situ hybridization to analyse the effects of short-term (1 week) or long-term (4 months) OVx on cerebral NF-L mRNA expression. We focussed on the basal forebrain and the major targets of its projections, hippocampus and cortex, since these regions play a role in cognition. Using in situ hybridization, we also investigated whether overall changes in NF-L mRNA levels were due to alterations in expression involving sub-populations of medial septal neurons or CA1 hippocampal neurons.

\section{EXPERIMENTAL PROCEDURES}

\section{Animals and surgery}

Female Fischer 344 rats (retired breeders) were obtained at the age of nine to 10 months from a single colony (Harlan Sprague Dawley, Indianapolis, IN). Animals were housed two per cage in an approved animal care facility with water and food provided $a d$ libitum for at least two weeks prior to the performance of any surgical procedures. All efforts were made to minimize both the suffering and number of animals used and all experiments were approved by the McGill University animal care utilization committee and carried out in accordance with the requirements of the Canadian Council on Animal Care.

Vaginal examinations and cytological studies were performed in all animals prior to the surgery in order to co-ordinate its timing within the estrous cycle. Following the onset of anesthesia with ketamine $(8.5 \mathrm{mg} / 100 \mathrm{~g})$ and xylazine $(0.3 \mathrm{mg} / 100 \mathrm{~g})$ i.m., either sham or bilateral OVx surgery was performed using a posterior surgical approach. Complete surgical castration was ensured by the visible appearance of vaginal mucosal atrophy as well as a vaginal cytological smear exhibiting the absence of mature epithelial cells. Vaginal smears were performed on all animals within 1 week after the surgery. The presence of mature cells on vaginal cytology in OVx animals was indicative of stimulation by estrogen from residual ovarian tissue and any such animals would normally be killed and completely excluded from further analysis. RNase protection assay was performed one week (short-term $\mathrm{OVx}$ ) or four months (long-term OVx) after surgery and in situ hybridization experiments were conducted only at the latter timepoint (i.e. four months after surgery). Vaginal examinations and cytological studies were performed in all animals during the week prior to death. None of the OVx animals demonstrated any evidence of estrogen stimulation, while as expected for 13-14month-old rats, most sham animals were in a state of constant estrus. The remaining sham animals demonstrated cyclical patterns and were killed during estrus.

\section{RNA extraction}

Following induction of deep anesthesia, rats (16 sham and 12 Ovx for each time-course) were decapitated. Their brains were rapidly removed and chilled. Specific cerebral regions (frontal and occipital cortex, hippocampus, hypothalamus and striatum) were dissected on ice and all tissues were then immediately snap frozen and kept at $-80^{\circ} \mathrm{C}$ until used. Total RNA was extracted according to the modified method of Chomczynski. ${ }^{9}$ Tissues were homogenized using glass/Teflon homogenizers in TRIzol reagent (Gibco BRL, Gaithersburg, MD). Following chloroform extraction, RNA contained in the aqueous phase was precipitated with isopropanol. Resulting pellets were then washed with $70 \%$ ethanol, air-dried, re-suspended in diethylpyrocarbonate (DEPC)treated $\mathrm{H}_{2} \mathrm{O}$ and stored at $-80^{\circ} \mathrm{C}$. Quantitation of the total RNA concentration in each individual sample was performed by spectrophotometry (UV absorbency at $260 \mathrm{~nm}$ and $280 \mathrm{~nm}$ ).

\section{Ribonuclease protection assay}

For RNase protection analysis a 349 base-pair fragment of the rat NF-L cDNA ${ }^{27}$ representing bases $125-474$ was subcloned into bluescript (Stratagene, La Jolla, CA). A ${ }^{32}$ P-labeled antisense RNA probe was prepared using $\mathrm{T}_{3}$ RNA polymerase (Promega, Madison, WI), under conditions recommended by the manufacturer. For internal control, a ${ }^{32} \mathrm{P}$-labeled RNA probe was prepared from a 316 base-pair fragment of glucose-6-phosphate dehydrogenase (GAPDH) cDNA (Ambion, Austin, TX). Riboprobes were treated with DNase 1 ( 1 unit/ $\mu \mathrm{g}$ DNA, $\left.37^{\circ} \mathrm{C}, 30 \mathrm{~min}\right)$. RNase protection assay was performed as described earlier. ${ }^{29}$ For each reaction, $5 \mu \mathrm{g}$ of total RNA was combined with $10^{8}$ c.p.m. of labeled NF-L and GAPDH probe, respectively, and allowed to hybridize overnight at $42^{\circ} \mathrm{C}$ in $90 \%$ formamide. Non-hybridized RNA was digested with RNase A $(40 \mu \mathrm{g} / \mathrm{ml})$ and RNase $\mathrm{T}_{1}(2 \mu \mathrm{g} / \mathrm{ml})$ for $1 \mathrm{~h}$ at $37^{\circ} \mathrm{C}$. RNases were deactivated by treatment with proteinase $\mathrm{K}(90 \mu \mathrm{g} /$ $\mathrm{ml}$ ) for $30 \mathrm{~min}$ at $37^{\circ} \mathrm{C}$. The protected RNA:RNA duplexes were electrophoresed on a non-denaturing $8 \%$ polyacrylamide gel at $200 \mathrm{~V}$ over $4 \mathrm{~h}$ in order to produce a satisfactory resolution of the NF-L and GADPH bands. The gel was dried and exposed for $2 \mathrm{~h}$ on a phosphoimager screen. Intensity of chemoluminescent bands was measured using a phosphoimager system (Fuji BAS 2000). Levels of NF-L mRNA were normalized by expressing them as a ratio of NF-L mRNA/GAPDH mRNA. Linearity of assay was assured by the development of standard curves using both varying amounts of total RNA and known quantities of synthetic sense NF-L RNA (data not shown).

\section{In situ hybridization}

Following anesthesia and decapitation, brains were removed from rats (three sham and three OVx). The two hemispheres were separated and two complementary hemibrains, one from a sham 
animal and the other from an ovariectomized rat, were jointly embedded in cold Tissue-Tek medium (Miles Laboratory, Tarrytown, NY). They were then frozen at $-55^{\circ} \mathrm{C}$ in 2-methyl butane and stored at $-80^{\circ} \mathrm{C}$ until further use. Sections $(10 \mu \mathrm{m})$ were cut from frozen tissue using a cryostat and were mounted on slides (Probe-on, Fisher Scientific). They were hybridized overnight at $42^{\circ} \mathrm{C}$ in a humidified chamber with a solution containing $80 \mathrm{ng}$ of sense or antisense oligonucleotide probes labeled with ${ }^{33} \mathrm{P}-\mathrm{dATP}(3000 \mathrm{Ci} / \mathrm{mmol}$, NEG-312H, New England Nuclear, Boston, MA); $1 \mathrm{Ci}=37 \mathrm{GBq}$ ). Hybridization buffer contained $50 \%$ formamide, saline sodium citrate (SSC) $4 \times$, dextran sulfate $(100 \mathrm{mg} / \mathrm{ml})$, sarcosyl $(1 \%)$, Denhardt's solution $(1 \times)$, salmon sperm DNA $(6 \mathrm{mM})$ and dithiothreitol $(6 \mathrm{mM})$. Sense and antisense oligonucleotides were labeled using terminal transferase reaction. ${ }^{49}$ After hybridization, sections were washed four times at $55^{\circ} \mathrm{C}$ for $15 \mathrm{~min}$ and once at room temperature for $30 \mathrm{~min}$ in SSC $1 \times$. The slides were washed in deionized water and dehydrated in $65 \%, 95 \%$ and $100 \%$ ethanol solution. Finally, slides were dried, dipped in emulsion (NTB2 Kodak, 1:1 with distilled water) and exposed for five days, after which they were developed, stained with $0.002 \%$ Toluidine Blue, and finally mounted with Permount and a coverslip. Anatomical identification of adult brain structures was based on the atlas of Paxinos and Watson.

\section{Image analysis (in situ hybridization)}

Quantitative measurements were performed on images captured with a cooled-CCD camera (Star I, Photometrics) from a sham and an ovariectomized hemibrain mounted on the same slide. All measurable cells with visible nucleus and cytoplasm were selected for analysis (total number of neurons examined in control/ovariectomized hemibrains: medial septum, $n=204 / 231$; CA1 region of hippocampus, $n=590 / 436$ ). Densities of grains present over the cellular cytoplasm were determined using dedicated software (ImagePro, Media Cybernetic). Grain density was corrected by subtracting background values and expressed as a labeling index (LI) defined by the ratio of grain density over the background.

\section{Statistical analysis}

For RNAse protection assay, the differences between the average of NF-L:GAPDH mRNA ratio in OVx vs sham group were statistically analysed using a one-way or two-way analysis of variance. For in situ hybridization studies the LI values of neuronal cells exhibiting a significant signal (i.e. $\mathrm{LI}>1$ ) were compared between neurons in sham and ovariectomized animals using a Mann-Whitney rank sum test and a chi-square analysis. In all instances $P<0.05$ was considered statistically significant.

\section{RESULTS}

\section{Animals}

All ovariectomized animals demonstrated a complete absence of mature vaginal cells at these time-points, while cytological smears from most sham-operated animals exhibited constant estrus, a pattern typical for animals of this age. ${ }^{32}$ Completeness of bilateral OVx was also confirmed by a post mortem examination where all $\mathrm{OVx}$ animals included in our study exhibited profound uterine atrophy. As expected, bilateral OVx surgery was associated with a significant gain in body weight $(275 \pm 30 \mathrm{~g}$ vs $305 \pm 25 \mathrm{~g} ; P<0.05)$ and uterine weights decreased significantly $(758 \pm 117 \mathrm{mg}$ vs $247 \pm 89 \mathrm{mg} ; P<0.05)$.

\section{Ribonuclease protection assay}

We evaluated the impact of both short-term (1 week) and long-term (4 months) OVx on NF-L mRNA expression in five discrete brain regions. The hypothalamus was selected because of its known responsiveness to estrogen, while the striatum was chosen in view of its relative stability in AD. The septum was not dissected for RNAse protection studies in view of its proximity to other relevant structures and the inevitable presence of non-septal neurons in dissections obtained using standard techniques. However, three regions that are known targets of basal forebrain projections were studied (hippocampus, frontal cortex and occipital cortex). In order to see whether acute deprivation of GS-induced changes in NF-L mRNA expression, we measured levels of NF-L mRNA on gel bands (Fig. 1) corrected for GAPDH mRNA after short-term OVx. NF-L mRNA: GAPDH mRNA ratio varied somewhat in the different regions examined and ranged from $\sim 0.30$ (hypothalamus) to $\sim 0.70$ (striatum, hippocampus, cortex) in sham animals. Following short-term OVx, there were no significant or consistent changes in NF-L mRNA expression in any of the regions examined (Figs 2, 3). Although there was a non-significant trend for decreased NF-L mRNA expression (up to $-8.8 \%$ ) in the striatum, frontal cortex and hippocampus after OVx, there was a trend in the opposite direction in the hypothalamus and occipital cortex (up to $+9 \%$ ). We subsequently repeated these analyses after a long-term OVx to determine which of these tendencies may be enhanced by chronic GS deprivation. NF-L mRNA/GAPDH mRNA levels were significantly increased after long-term OVx in the hippocampus, frontal cortex and occipital cortex (14.9\%, $+12 \%$ and $+8.8 \%$ respectively; $P<0.02$; Fig. 3). Changes in striatum $(-4 \%)$ and hypothalamus $(+11.8 \%)$ were not significant (Fig. 2).

\section{In situ hybridization}

In situ hybridization studies were conducted in order to determine whether any changes in NF-L mRNA expression could be observed in medial septal neurons. Another goal was to see whether some of the modest increase in overall NF-L mRNA levels in the septal targets could be localized to any particular subset of hippocampal neurons. The pyramidal layer of the CA1 field of the hippocampus was of particular interest, since many estrogen-mediated morphological changes have been described in this subregion. ${ }^{16}$ Figure 4 represents a photomicrograph with cells labeled by NF-L antisense probe in both structures examined. The majority of cells both in the medial septum and the CA1 region of the hippocampus demonstrated a modest expression of NF-L mRNA (Figs 5, 6), while some expressed NF-L mRNA at levels near that of the background. Most hippocampal cells demonstrating low NF-L mRNA expression (i.e. $\mathrm{LI} \leq 1$ ) tended to be smaller with an

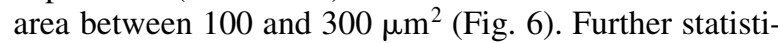
cal analyses were performed on cells with a significant level of NF-L expression (i.e. LI $>1$ ). The mean value of LI in this case was significantly greater in the $\mathrm{OVx}$ group compared to sham animals in the septum $(14.5 \pm 2.5$ vs $8.0 \pm 0.7, P<0.01)$, but not in the CA1 region of the hippocampus $(8.2 \pm 0.4$ vs $8.1 \pm 0.3)$. The proportion of neurons demonstrating a significant 


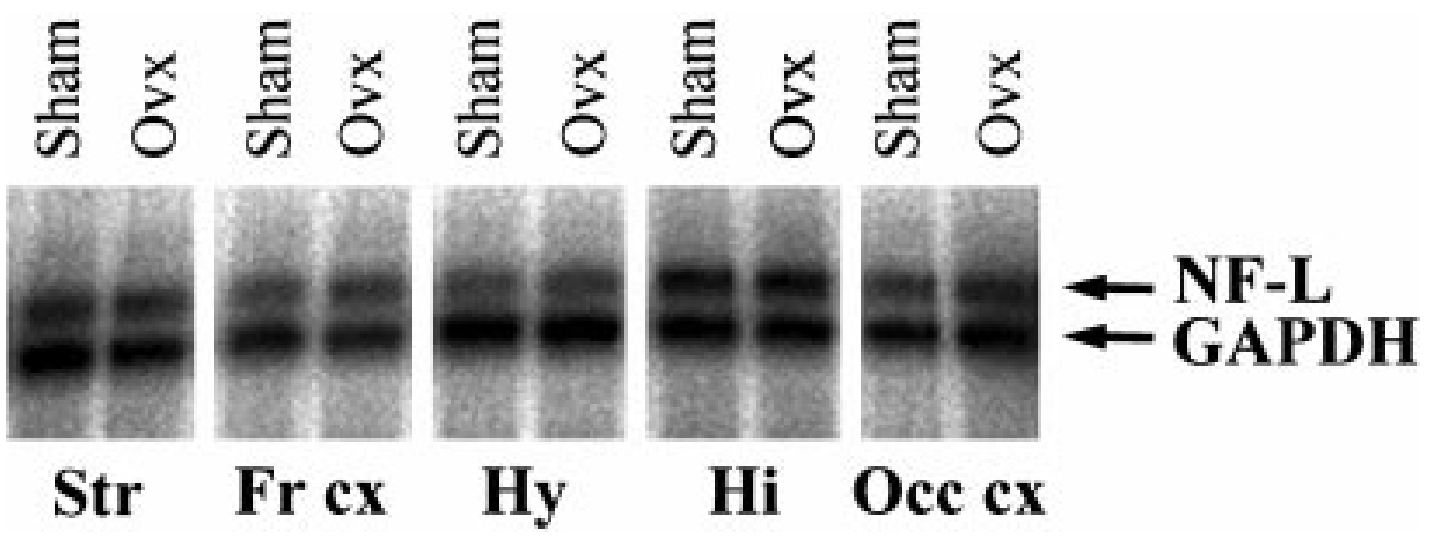

Fig. 1. Representative gel from one sham and one OVx animal showing the effect of OVx on NF-L (upper band, 349 bp) and GAPDH (lower band, $316 \mathrm{bp}$ ) protected RNA:RNA duplexes. Levels of NF-L mRNA were evaluated by phosphoimaging and normalized by expressing them as a ratio of NF-L:GAPDH mRNA signal. Str, striatum; Fr cx, frontal cortex; Hy, hypothalamus; Hi, hippocampus; Occ cx, occipital cortex.

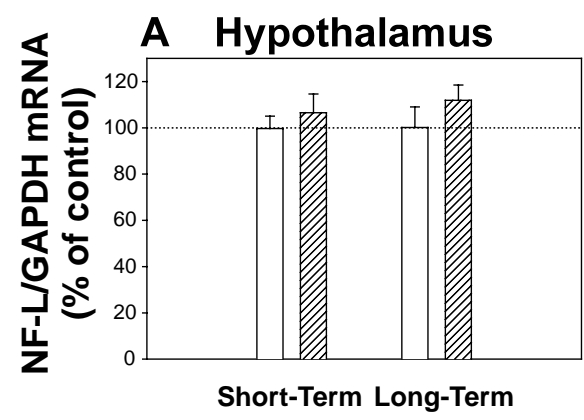

$\square$ Sham

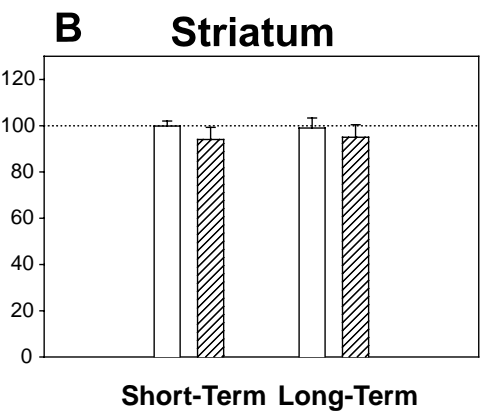

DID OVx

Fig. 2. Measurement (expressed as mean percentage of controls \pm S.E.M) of NF-L mRNA changes induced by long-term or shortterm OVx in control areas, i.e. hypothalamus (A) and striatum (B). There was no significant change in NF-L level following either short- or long-term OVx.
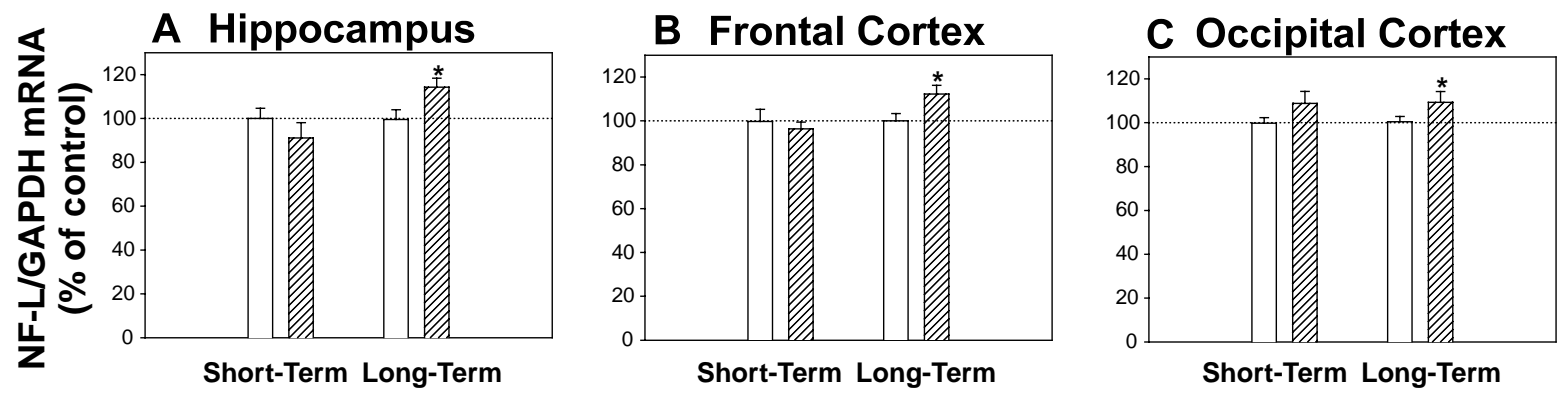

$\square$ Sham $\quad$ m OVx

Fig. 3. Measurement (expressed as mean percentage of controls \pm S.E.M) of NF-L mRNA changes induced by long-term or shortterm OVx in brain areas related to cognition, i.e. hippocampus (A), frontal cortex (B) and occipital cortex (C). Ratios were increased in ovariectomized animals $(n=12)$ compared with sham $(n=16)$ only following long-term OVx $(P<0.05)$.

NF-L mRNA expression (i.e. $\mathrm{LI}>1$ ) in the medial septum did not change after OVx (sham vs OVx; 78\% vs $74 \%$; NS). Although, the proportion of CA1 hippocampal neurons with LI $>1$ appeared to increase with OVx, this difference did not reach statistical significance (sham vs OVx; $88 \%$ vs $96 \% ; P=0.06$, NS). In the medial septum, a population of small neurons (50-250 $\mu^{2}$ ) expressed much higher NF-L mRNA expression (LI > 6) after long-term OVx (Fig. 5). Following longterm $\mathrm{OVx}$ there also appeared to be an increased number

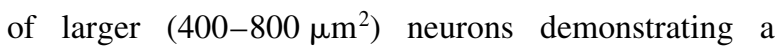
moderate up-regulation of NF long-term OVx (Fig. 6).

\section{DISCUSSION}

Our results provide quantitative evidence that NF-L mRNA expression is increased in specific brain regions following long-term, but not short-term, OVx. As discussed below, we propose that this increase in NF-L mRNA expression could be reflective of an axonal 

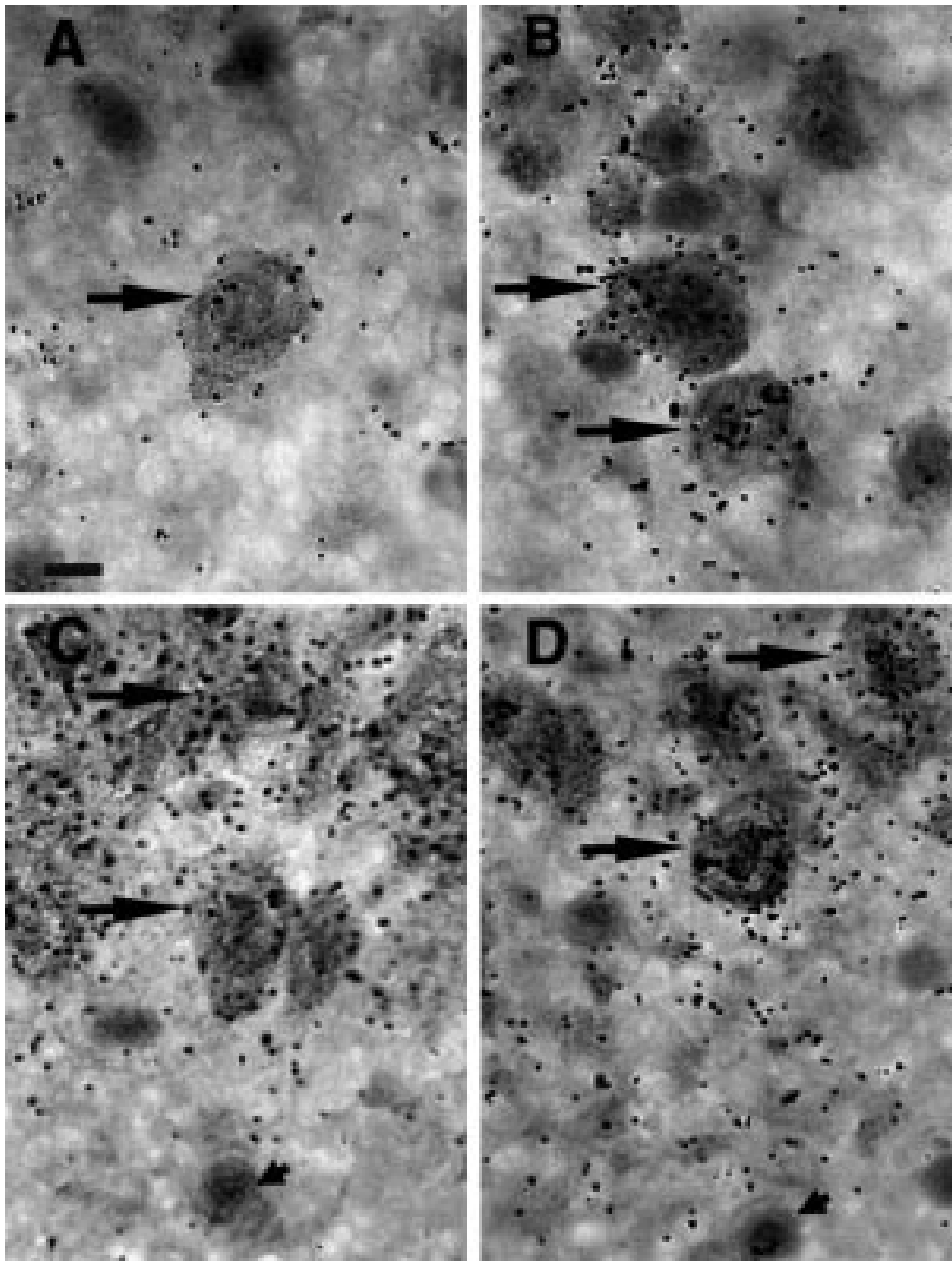

Fig. 4. NF-L mRNA in situ hybridization. Representative images are presented from medial septal (A, B) and CA1 hippocampal (C, D) regions in sections obtained from animals which had undergone sham $(\mathrm{A}, \mathrm{C} ; n=3)$ or bilateral $\mathrm{OVx}(\mathrm{B}, \mathrm{D} ; n=3)$ surgery four months earlier. Large arrows (A-D) highlight neurons which express NF-L mRNA, demonstrating increased expression in some septal neurons after bilateral OVx (top arrow in B). Small arrows indicate the presence of smaller hippocampal neurons which express very low (i.e. non-specific) NF-L mRNA levels. Scale bar $=10 \mu \mathrm{m}$.

reorganization that has taken place in order to compensate for a loss of GS trophic support for certain categories of responsive neurons.

\section{Regional and temporal specificity of the effects induced by ovariectomy}

Neurofilament proteins are a major cytoskeletal constituent and play an important role in both the attainment and maintenance of normal neuronal structure. In this study, we examined the effects of both short- and long-term OVx on NF-L mRNA expression in several rat brain regions. OVx has been shown to result in a number of atrophic changes. These include alterations in a variety of intracytoplasmic organelles such as the endoplasmic reticulum, mitochondria and lysosomes, as well as depletion of microtubules and filaments in dendritic processes. ${ }^{7}$ In addition, GS (particularly estrogen) concentration reduction provoke dendritic spines withdrawal in both hippocampal ${ }^{16,40}$ and septal ${ }^{38}$ neuronal cultures. NF-L mRNA levels are reduced in early axonal degeneration, ${ }^{28}$ in aging, ${ }^{46}$ as well as in several neurodegenerative diseases. ${ }^{25}$ As a result, we expected that the atrophic effect of OVx would result in decreased NF-L mRNA expression, particularly in the brain regions known to be highly sensitive to estrogen. Contrary to these expectations, in our studies, short-term (one week) OVx did not appear to have a significant effect on NF-L mRNA expression in any of the brain regions studied, including the hypothalamus, which is known to be estrogen sensitive. However, when a much later time-point was evaluated, data obtained using RNAse protection measurements indicated modest but significant increases in NF-L mRNA expression involving the hippocampus, the frontal cortex and occipital cortex, but not the hypothalamus or the striatum. In situ hybridization evaluation of the medial septum also demonstrated increases in NF-L mRNA expression. We performed both 


\section{MEDIAL SEPTUM}
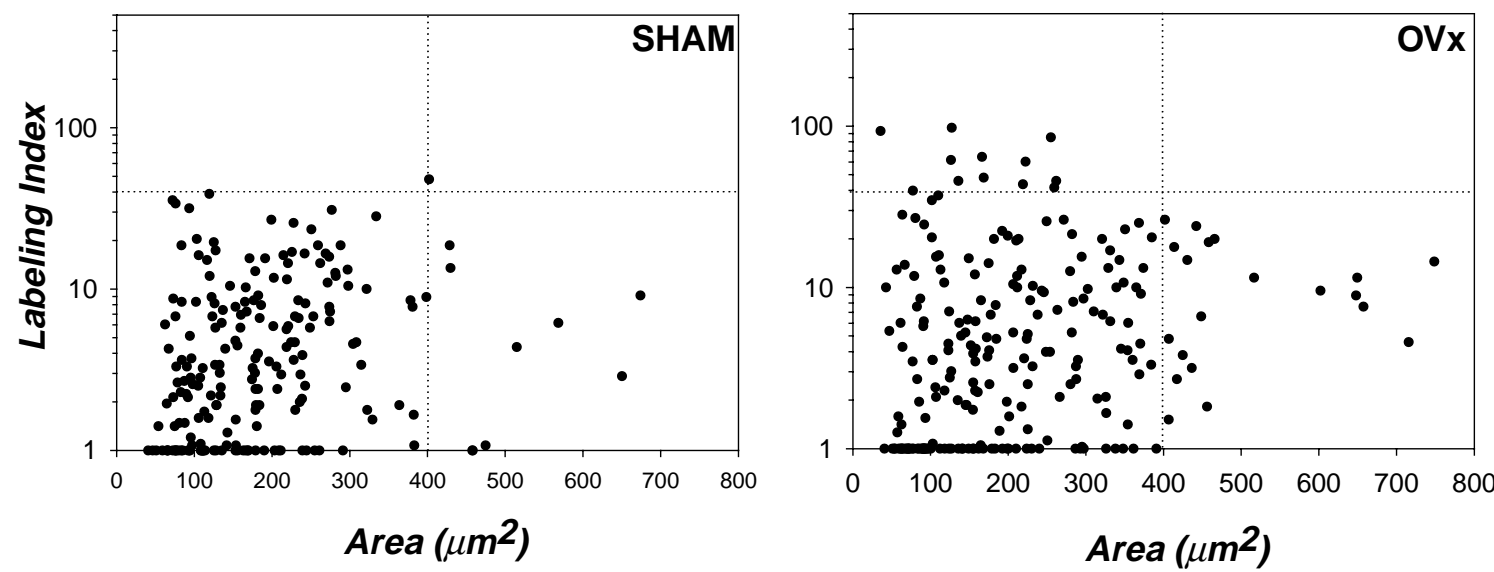

Fig. 5. Distribution of LI (expressed on a logarithmic scale) as a function of the cytoplasmic area of cells in the medial septum of sham (left panel, $n=3$ ) or ovariectomized (right panel, $n=3$ ) animals. Each dot represents one cell counted in either of two hemisections, one from sham, one from ovariectomized groups processed through in situ hybridization together on the same slide. A population of small neurons expressed high levels of NF-L mRNA after long-term OVx (upper left quadrant).

\section{HIPPOCAMPUS (CA1)}

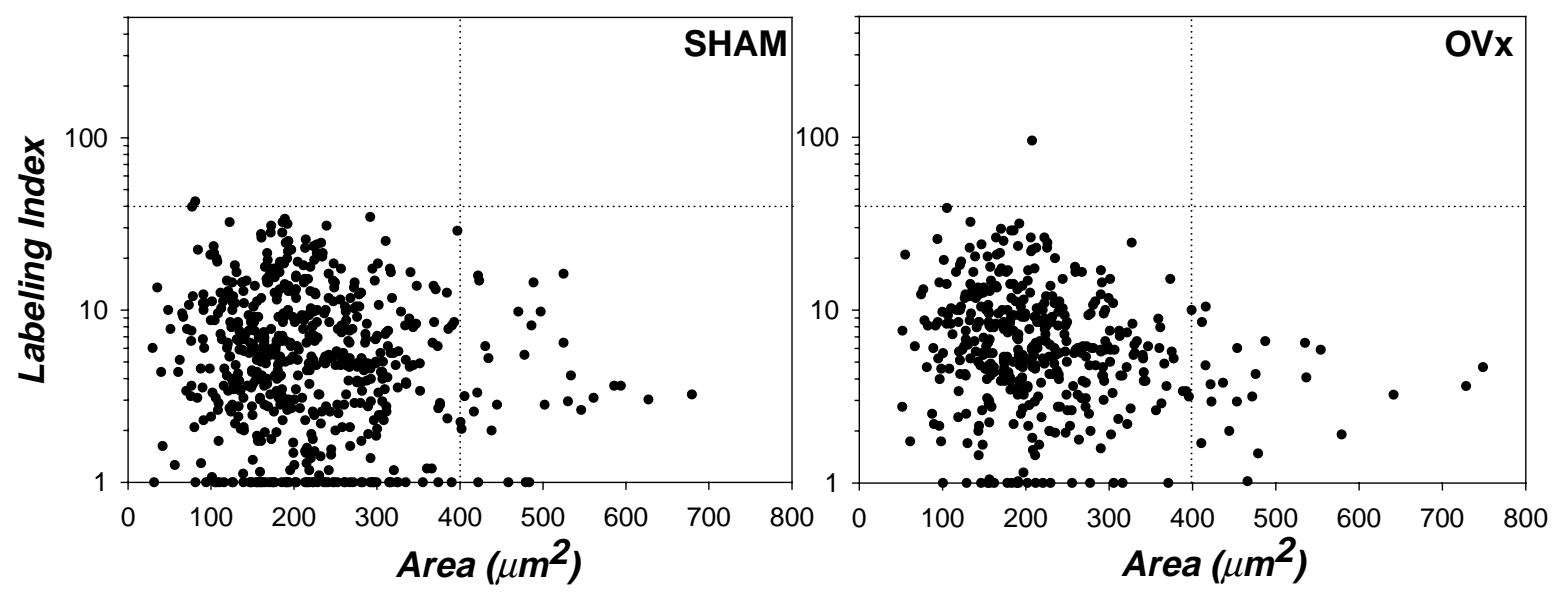

Fig. 6. Distribution of LI (expressed on a logarithmic scale) as function of the cytoplasmic area of cells in the CA1 pyramidal layer of the hippocampus of sham (left panel, $n=3$ ) or ovariectomized (right panel, $n=3$ ) animals. Each dot represents one cell counted in either of two hemisections, one from sham, one from ovariectomized groups processed for in situ hybridization together on the same slide. There appeared to be fewer neurons expressing low levels of NF-L mRNA $(\mathrm{LI}<1)$ after long-term OVx $(x$-axis).

RNAse protection and in situ hybridization NF-L mRNA measurements in the hippocampus. Interestingly, while NF-L mRNA expression increased in the former study after long-term OVx, it did not change in the latter. This difference can be attributed to the fact that the in situ study focussed on the pyramidal layer of the CA1 region, while mRNA was extracted from the entire hippocampus for RNAse protection measurements. In addition, the ability of the two techniques to quantify small differences in mRNA expression varies.

Our results first indicate that the effects of GS deprivation on NF-L expression are delayed. The differential effects of $\mathrm{OVx}$ with time have been previously reported in other systems where long-term, but not short-term, OVx induced changes in choline acetyltransferase (ChAT), the tyrosine kinase receptor trkA, ${ }^{36}$ nerve growth factor (NGF), brain-derived factor ${ }^{54}$ and dopamine transporter ${ }^{4}$ mRNA expression. Thus, the delayed impact of GS deficit on NF-L mRNA expression is by no means unique or specific for this gene, supporting the concept that secondary compensatory mechanisms are likely to play a role in these events.

Our findings also emphasize the profound effects of GS deficit on basal forebrain and related regions, which are the anatomical substrate for cholinergic neurotransmission. Interestingly, cholinergic neuron functioning is impaired by OVx, as demonstrated by numerous studies showing decreases in ChAT activity, ${ }^{61}$ choline uptake ${ }^{43}$ and acetylcholine release, ${ }^{15}$ as well as diminished ChAT and trkA mRNA expression. ${ }^{14,36}$ Recent studies propose that this impairment may result from a synergistic interaction between estrogen and neurotrophin pathways (see Ref. 55), as basal forebrain cholinergic neurons specifically express NGF receptors and show high-affinity 
estrogen binding sites $^{59}$ and estrogen $\alpha$-receptor-like immunoreactivity. ${ }^{13,37}$ Thus, the vulnerability of cholinergic neurons to $\mathrm{OVx}$ due to alteration of trophic support provides support for our observations demonstrating that long-term OVx has a particularly significant effect on NF-L mRNA expression in basal forebrain neurons, as well as in neurons in areas receiving basal forebrain projections. In situ hybridization studies demonstrated that increased NF-L mRNA expression was occurring in a subpopulation of individual neurons in the medial septum. Although there were no significant changes in NF-L mRNA expression in the pyramidal layer of the CA1 region of the hippocampus, overall hippocampal expression of NF-L mRNA increased. The increases in NF-L mRNA expression following long-term OVx may be taking place in other hippocampal cells such as the ones located in the dentate gyrus or the CA2-3 fields. Alternatively, it is also possible that small neurons which normally express very low levels of NF-L mRNA demonstrate small increases in this expression following OVx, increases which may have been difficult to detect using in situ hybridization. Our data indicate the possibility that long-term $\mathrm{OVx}$ increases the proportion of CA1 neurons which express significant NF-L mRNA $(\mathrm{LI}>1)$. These latter cells may be local neurons with short axonal process, since neuronal neurofilament expression appears to correlate with, and possibly determine, the size ${ }^{51}$ and caliber ${ }^{20}$ of individual axons. Based on these considerations, it could be proposed that $\mathrm{OVx}$ first induces morphological alterations including axonal atrophy involving basal forebrain neurons ${ }^{13}$ and that this deafferentation of projection areas is followed by compensatory sprouting reflected by NF-L mRNA upregulation. Such reorganization of the terminal arborization could be taking place both in "intact" basal forebrain neurons projecting to these targets, as well as in local neurons in these target regions.

\section{Relevance of increased neurofilament mRNA expression to cytoskeletal changes}

It is not entirely clear whether NF-L plays an active role in either neuronal regeneration or sprouting. In favor of the proposal that NF-L plays a role in axonal growth are studies showing that neurite outgrowth in cultured cells is associated with NF-L up-regulation. ${ }^{58}$ However, a lack of NF-L in NF-L knock-out mice does not prevent axonal regrowth after a sciatic nerve crush injury but introduces a delay in maturation of axons. ${ }^{63}$ In contrast, NF-L mRNA levels are decreased in the dorsal root ganglion in the early phase of axonal injury following a sciatic nerve lesion in rats. ${ }^{22,29}$ This reduction is reversed after 12 weeks of nerve regrowth ${ }^{23}$ suggesting that NF-L is more important in the late, than the immediate, phase of axonal regrowth. Consistently, the lack of change in NF-L expression after short-term OVx demonstrated here indicates that NF-L is probably not required for immediate sprouting. Based on these considerations, we propose that the increased NF-L mRNA levels in long-term OVx reflect previous axonal collateral sprouting in neurons now required to sustain larger and more complex axonal projections. This proposal concurs with a role for neurofilaments in the consolidation of previous neuritic growth through either a stabilization of microtubules and actin filament dynamics or the creation of an axonal scaffold (for reviews, see Refs 25 and 26). An interesting alternative hypothesis regarding the role of neurofilaments in axonal growth has been suggested by Gupta and colleagues. They proposed that up-regulation of neurofilament expression is the actual rescue mechanism which prevents or delays neurodegeneration ${ }^{17}$ based on a correlation between good preservation of neuronal morphology and the level of neurofilament overexpression after induced-delayed neurotoxicity. This hypothesis is consistent with our results, since increased NF-L mRNA levels could be reflective of the induction of such a protective effect taking place in response to the loss of trophic support, as a result of diminished GS levels.

\section{Mechanisms of ovariectomy effects on neurofilament mRNA levels}

Whether or not NF-L mRNA expression is under the direct control of GS is not known. OVx results in a deficit of estrogen and rodent NF-L promoter possesses both AP-1 and sp1 sites, ${ }^{48,41}$ which are both part of the estrogen responsive element. ${ }^{45}$ AP-1 transcription factors such as fos and jun can be induced by estrogen in vivo ${ }^{8}$ and are able to enhance NF-L expression in culture. ${ }^{48}$ However, the lack of major changes in NF-L mRNA expression after short-term OVx does not support the presence of a strong estrogenic control of the NF-L promoter in mature rats. Moreover, there is no clearcut correlation between topographic brain distribution of the regions which demonstrated increased NF-L mRNA expression and the ones able to bind estrogen ${ }^{47}$ or express estrogen receptors. ${ }^{52}$ The hypothalamus, in particular, is highly receptive to estrogen, but OVx had virtually no effect on NF-L mRNA expression in this region. In contrast, changes in NF-L mRNA levels in the cerebral neocortex and hippocampus are quite widespread, although estrogen receptors are only expressed modestly in discrete neuronal subpopulations of these regions. ${ }^{52}$

Indirect effects of OVx on NF-L mRNA levels could be exerted by intermediary agents known to increase NF-L expression. For example, both $\mathrm{NGF}^{30,21}$ and somatostatin $^{58}$ are able to enhance NF-L expression in vitro. Interestingly, both $\mathrm{NGF}^{2}$ and somatostatin ${ }^{39}$ content is increased after cholinergic deficit in the hippocampus and cortex and OVx is known to induce cholinergic deficit in these regions. A hypothetical scenario for increased NF-L expression after OVx may involve these proteins. To further establish a parallel with our finding, NGF has been suggested to promote neuritic reorganization of preserved neurons after cholinergic deafferentation. ${ }^{1,3}$ Future studies will be required to establish whether or not neurotrophin or related molecules can be implicated in $\mathrm{OVx}$-induced changes in neurofilament expression and cytoskeletal reorganization. 


\section{CONCLUSIONS}

To date, most studies examining the role of ovarian hormones on brain function have evaluated the impact of short-term OVx and the administration of exogenous estrogens, often in supraphysiological concentrations. In contrast, we showed that chronic, but not short-term, OVx induced up-regulation of NF-L mRNA which may be important to sustain stabilization of the axonal arborization at the level of the "partially" denervated targets of septal projections. These results suggest that the impact of the GS deficit on the brain is progressive and that the benefits of HRT on neural function may be dependent on the timing of its administration following onset of GS deficit.

Acknowledgements-This work was supported by MRC of Canada (J.-P. Julien and G. A.Kuchel). E. Vaucher was a recipient of a post-doctoral fellowship from subsequently le Fond de la Recherche en Santé du Québec and Alzheimer Society of Canada. P. Pierret received a post-doctoral fellowship from the Parkinson Foundation of Canada. The authors would like to thank Martin Beaulieu for careful reading the manuscript and helpful discussions.

\section{REFERENCES}

1. Andrews T. J. and Cowen T. (1994) In vivo infusion of NGF induces the organotypic regrowth of perivascular nerves following their atrophy in aged rats. J. Neurosci. 14, 3048-3058.

2. Arendt T., Bruckner M. K., Krell T., Pagliusi S., Kruska L. and Heumann R. (1995) Degeneration of rat cholinergic basal forebrain neurons and reactive changes in nerve growth factor expression after chronic neurotoxic injury-II. Reactive expression of the nerve growth factor gene in astrocytes. Neuroscience 65, 647-659.

3. Arendt T., Bruckner M. K., Pagliusi S. and Krell T. (1995) Degeneration of rat cholinergic basal forebrain neurons and reactive changes in nerve growth factor expression after chronic neurotoxic injury-I. Degeneration and plastic response of basal forebrain neurons. Neuroscience $\mathbf{6 5}$, 633-645.

4. Bosse R., Rivest R. and Di Paolo T. (1997) Ovariectomy and estradiol treatment affect the dopamine transporter and its gene expression in the rat brain. Brain Res. Mol. Brain Res. 46, 343-346.

5. Brinton R. D., Tran J., Proffitt P. and Montoya M. (1997) 17 beta-Estradiol enhances the outgrowth and survival of neocortical neurons in culture. Neurochem. Res. 22, 1339-1351.

6. Cambiasso M. J., Diaz H., Caceres A. and Carrer H. F. (1995) Neuritogenic effect of estradiol on rat ventromedial hypothalamic neurons cocultured with homotopic or heterotopic glia. J. Neurosci. Res. 42, 700-709.

7. Carrer H. F. and Aoki A. (1982) Ultrastructural changes in the hypothalamic ventromedial nucleus of ovariectomized rats after estrogen treatment. Brain Res. 240, 221-233.

8. Cattaneo E. and Maggi A. (1990) c-fos induction by estrogen in specific rat brain areas. Eur. J. Pharmac. 188, 153-159.

9. Chomczynski P. and Sacchi N. (1987) Single-step method of RNA isolation by acid guanidinium thiocyanate- phenol-chloroform extraction. Analyt. Biochem. 162, 156-159.

10. Clark A. W., Krekoski C. A., Parhad I. M., Liston D., Julien J. P. and Hoar D. I. (1989) Altered expression of genes for amyloid and cytoskeletal proteins in Alzheimer cortex. Ann. Neurol. 25, 331-339.

11. Dickson T. C., King C. E., McCormack G. H. and Vickers J. C. (1999) Neurochemical diversity of dystrophic neurites in the early and late stages of Alzheimer's disease. Expl Neurol. 156, 100-110.

12. Drake E. B., Henderson V. W., Stanczyk F. Z., McCleary C. A., Brown W. S., Smith C. A., Rizzo A. A., Murdock G. A. and Buckwalter J. G. (2000) Associations between circulating sex steroid hormones and cognition in normal elderly women. Neurology 54, 599-603.

13. Gibbs R. B. (1996) Expression of estrogen receptor-like immunoreactivity by different subgroups of basal forebrain cholinergic neurons in gonadectomized male and female rats. Brain Res. 720, 61-68.

14. Gibbs R. B. (1998) Levels of trkA and BDNF mRNA, but not NGF mRNA, fluctuate across the estrous cycle and increase in response to acute hormone replacement. Brain Res. 787, 259-268.

15. Gibbs R. B., Hashash A. and Johnson D. A. (1997) Effects of estrogen on potassium-stimulated acetylcholine release in the hippocampus and overlying cortex of adult rats. Brain Res. 749, 143-146.

16. Gould E., Woolley C. S., Frankfurt M. and McEwen B. S. (1990) Gonadal steroids regulate dendritic spine density in hippocampal pyramidal cells in adulthood. J. Neurosci. 10, 1286-1291.

17. Gupta R. P., Lin W. W. and Abou-Donia M. B. (1999) Enhanced mRNA expression of neurofilament subunits in the brain and spinal cord of diisopropyl phosphorofluoridate-treated hens. Biochem. Pharmac. 57, 1245-1251.

18. Henderson V. W. (1997) Estrogen, cognition, and a woman's risk of Alzheimer's disease. Am. J. Med. 103, 11S-18S.

19. Hof P. R., Cox K. and Morrison J. H. (1990) Quantitative analysis of a vulnerable subset of pyramidal neurons in Alzheimer's disease: I. Superior frontal and inferior temporal cortex. J. comp. Neurol. 301, 44-54.

20. Hoffman P. N., Cleveland D. W., Griffin J. W., Landes P. W., Cowan N. J. and Price D. L. (1987) Neurofilament gene expression: a major determinant of axonal caliber. Proc. natn. Acad. Sci. U.S.A. 84, 3472-3476.

21. Ikenaka K., Nakahira K., Takayama C., Wada K., Hatanaka H. and Mikoshiba K. (1990) Nerve growth factor rapidly induces expression of the 68-kDa neurofilament gene by post-transcriptional modification in PC12h-R cells. J. biol. Chem. 265, 19,782-19,785.

22. Jiang Y. Q., Pickett J. and Oblinger M. M. (1994) Comparison of changes in beta-tubulin and NF gene expression in rat DRG neurons under regeneration-permissive and regeneration-prohibitive conditions. Brain Res. 637, $233-241$.

23. Jiang Y. Q., Pickett J. and Oblinger M. M. (1994) Long-term effects of axotomy on beta-tubulin and NF gene expression in rat DRG neurons. J. neural Transplant Plast. 5, 103-114.

24. Judd H. L. and Fournet N. (1994) Changes of ovarian hormonal function with aging. Expl Gerontol. 29, 285-298.

25. Julien J. P. (1999) Neurofilament functions in health and disease. Curr. Opin. Neurobiol. 9, 554-560.

26. Julien J. P. and Mushynski W. E. (1998) Neurofilaments in health and disease. Prog. Nucleic Acid Res. Mol. Biol. 61, 1-23.

27. Julien J. P., Ramachandran K. and Grosveld F. (1985) Cloning of a cDNA encoding the smallest neurofilament protein from the rat. Biochim. biophys. Acta 825, 398-404.

28. Kuchel G. A., Poon T., Irshad K., Richard C., Julien J. P. and Cowen T. (1997) Decreased neurofilament gene expression is an index of selective axonal hypotrophy in ageing. NeuroReport 8, 799-805.

29. Kuchel G. A., Rowe W., Meaney M. J. and Richard C. (1997) Neurotrophin receptor and tyrosine hydroxylase gene expression in aged sympathetic neurons. Neurobiol. Aging 18, 67-79.

30. Lindenbaum M. H., Carbonetto S. and Mushynski W. E. (1987) Nerve growth factor enhances the synthesis, phosphorylation, and metabolic stability of neurofilament proteins in PC12 cells. J. biol. Chem. 262, 605-610. 
31. Lorenzo A., Diaz H., Carrer H. and Caceres A. (1992) Amygdala neurons in vitro: neurite growth and effects of estradiol. J. Neurosci. Res. 33, $418-435$

32. Lu K. H., Hopper B. R., Vargo T. M. and Yen S. S. (1979) Chronological changes in sex steroid, gonadotropin and prolactin secretions in aging female rats displaying different reproductive states. Biol. Reprod. 21, 193-203.

33. Luine V. N. (1985) Estradiol increases choline acetyltransferase activity in specific basal forebrain nuclei and projection areas of female rats. Expl Neurol. 89, 484-490.

34. Ma D., Descarries L., Micheva K. D., Lepage Y., Julien J. P. and Doucet G. (1999) Severe neuronal losses with age in the parietal cortex and ventrobasal thalamus of mice transgenic for the human NF-L neurofilament protein. J. comp. Neurol. 406, 433-448.

35. McLachlan D. R., Lukiw W. J., Wong L., Bergeron C. and Bech-Hansen N. T. (1988) Selective messenger RNA reduction in Alzheimer's disease. Brain Res. 427, 255-261.

36. McMillan P. J., Singer C. A. and Dorsa D. M. (1996) The effects of ovariectomy and estrogen replacement on trkA and choline acetyltransferase mRNA expression in the basal forebrain of the adult female Sprague-Dawley rat. J. Neurosci. 16, 1860-1865.

37. Miller M. M., Hyder S. M., Assayag R., Panarella S. R., Tousignant P. and Franklin K. B. (1999) Estrogen modulates spontaneous alternation and the cholinergic phenotype in the basal forebrain. Neuroscience 91, 1143-1153.

38. Miyakawa M. and Arai Y. (1987) Synaptic plasticity to estrogen in the lateral septum of the adult male and female rats. Brain Res. 436, $184-188$.

39. Moyse E., Szigethy E., Danger J. M., Vaudry H., Wenk G. L., Beaudet A. and Epelbaum J. (1993) Short- and long-term effects of nucleus basalis magnocellularis lesions on cortical levels of somatostatin and its receptors in the rat. Brain Res. 607, 154-160.

40. Murphy D. D. and Segal M. (1996) Regulation of dendritic spine density in cultured rat hippocampal neurons by steroid hormones. J. Neurosci. 16, 4059-4068.

41. Nakahira K., Ikenaka K., Wada K., Tamura T., Furuichi T. and Mikoshiba K. (1990) Structure of the 68-kDa neurofilament gene and regulation of its expression. J. biol. Chem. 265, 19,786-19,791.

42. Nakamura Y., Hasimoto R., Kashiwagi Y., Miyamae Y., Shinosaki K., Nishikawa T., Hattori H., Kudo T. and Takeda M. (1997) Abnormal distribution of neurofilament L in neurons with Alzheimer's disease. Neurosci. Lett. 225, 201-204.

43. O'Malley C. A., Hautamaki R. D., Kelley M. and Meyer E. M. (1987) Effects of ovariectomy and estradiol benzoate on high-affinity choline uptake, ACh synthesis, and release from rat cerebral cortical synaptosomes. Brain Res. 403, 389-392.

44. Packard M. G. and Teather L. A. (1997) Intra-hippocampal estradiol infusion enhances memory in ovariectomized rats. NeuroReport 8, 3009-3013.

45. Paech K., Webb P., Kuiper G. G., Nilsson S., Gustafsson J., Kushner P. J. and Scanlan T. S. (1997) Differential ligand activation of estrogen receptors ERalpha and ERbeta at AP1 sites. Science 277, 1508-1510.

46. Parhad I. M., Scott J. N., Cellars L. A., Bains J. S., Krekoski C. A. and Clark A. W. (1995) Axonal atrophy in aging is associated with a decline in neurofilament gene expression. J. Neurosci. Res. 41, 355-366.

47. Pfaff D. and Keiner M. (1973) Atlas of estradiol-concentrating cells in the central nervous system of the female rat. J. comp. Neurol. 151, 121-158.

48. Pospelov V. A., Pospelova T. V. and Julien J. P. (1994) AP-1 and Krox-24 transcription factors activate the neurofilament light gene promoter in P19 embryonal carcinoma cells. Cell Growth Differ. 5, 187-196.

49. Sambrook J. (2000) In Molecular Cloning: A Laboratory Manual (eds Fritsch E. F and Maniatis T.). Cold Spring Harbor Laboratory Press, Cold Spring Harbor.

50. Schmidt M. L., Lee V. M. and Trojanowski J. Q. (1990) Relative abundance of tau and neurofilament epitopes in hippocampal neurofibrillary tangles. Am. J. Path. 136, 1069-1075.

51. Sharp G. A., Shaw G. and Weber K. (1982) Immunoelectronmicroscopical localization of the three neurofilament triplet proteins along neurofilaments of cultured dorsal root ganglion neurones. Expl Cell Res. 137, 403-413.

52. Simerly R. B., Chang C., Muramatsu M. and Swanson L. W. (1990) Distribution of androgen and estrogen receptor mRNA-containing cells in the rat brain: an in situ hybridization study. J. comp. Neurol. 294, 76-95.

53. Simpkins J. W., Green P. S., Gridley K. E., Singh M., de Fiebre N. C. and Rajakumar G. (1997) Role of estrogen replacement therapy in memory enhancement and the prevention of neuronal loss associated with Alzheimer's disease. Am. J. Med. 103, 19S-25S.

54. Singh M., Meyer E. M. and Simpkins J. W. (1995) The effect of ovariectomy and estradiol replacement on brain-derived neurotrophic factor messenger ribonucleic acid expression in cortical and hippocampal brain regions of female Sprague-Dawley rats. Endocrinology 136, $2320-2324$

55. Singh M., Setalo G. Jr, Guan X., Frail D. E. and Toran-Allerand C. D. (2000) Estrogen-induced activation of the mitogen-activated protein kinase cascade in the cerebral cortex of estrogen receptor-alpha knock-out mice. J. Neurosci. 20, 1694-1700.

56. Smith M. A., Rudnicka-Nawrot M., Richey P. L., Praprotnik D., Mulvihill P., Miller C. A., Sayre L. M. and Perry G. (1995) Carbonyl-related posttranslational modification of neurofilament protein in the neurofibrillary pathology of Alzheimer's disease. J. Neurochem. 64, $2660-2666$.

57. Su J. H., Cummings B. J. and Cotman C. W. (1996) Plaque biogenesis in brain aging and Alzheimer's disease. I. Progressive changes in phosphorylation states of paired helical filaments and neurofilaments. Brain Res. 739, 79-87.

58. Taniwaki T. and Schwartz J. P. (1995) Somatostatin enhances neurofilament expression and neurite outgrowth in cultured rat cerebellar granule cells. Brain Res. Dev. Brain Res. 88, 109-116.

59. Toran-Allerand C. D., Miranda R. C., Bentham W. D., Sohrabji F., Brown T. J., Hochberg R. B. and MacLusky N. J. (1992) Estrogen receptors colocalize with low-affinity nerve growth factor receptors in cholinergic neurons of the basal forebrain. Proc. natn. Acad. Sci. U.S.A. 89, $4668-4672$.

60. Vickers J. C., Riederer B. M., Marugg R. A., Buee-Scherrer V., Buee L., Delacourte A. and Morrison J. H. (1994) Alterations in neurofilament protein immunoreactivity in human hippocampal neurons related to normal aging and Alzheimer's disease. Neuroscience 62, 1-13.

61. Wu X., Glinn M. A., Ostrowski N. L., Su Y., Ni B., Cole H. W., Bryant H. U. and Paul S. M. (1999) Raloxifene and estradiol benzoate both fully restore hippocampal choline acetyltransferase activity in ovariectomized rats. Brain Res. 847, 98-104.

62. Xu Z., Cork L. C., Griffin J. W. and Cleveland D. W. (1993) Increased expression of neurofilament subunit NF-L produces morphological alterations that resemble the pathology of human motor neuron disease. Cell 73, 23-33.

63. Zhu Q., Couillard-Despres S. and Julien J. P. (1997) Delayed maturation of regenerating myelinated axons in mice lacking neurofilaments. Expl Neurol. 148, 299-316. 